\title{
Appreciation to Journal of Mathematical Imaging Reviewers
}

(C) Springer Science+Business Media New York 2015

For helping us deliver timely decisions to our authors, the Editor-in-Chief and Publisher would like to thank the following individuals that contributed reviews between January 1st, 2014 and December 31st, 2014. We applaud your efforts and dedication to the community.

Henrik Aanæs

Muhammad Abbas

Steffen Abraham

Manya Afonso

Gady Agam

Olivier Alata

Stéphanie Allassonnière

Mariana Almeida

L. Alvarez

Sergio Amat

Gaik Ambartsoumian

Yali Amit

Bjoern Andres

Jesus Angulo

Helder Araujo

Sabri Arik

Markus Aschwanden

Gilles Aubert

Jean-Francois Aujol

Didier Auroux

Tudor Barbu

Joao Barreto

Quentin Barthélemy

Joost Batenburg

Eliane Becache

Amir Beck

Saima Ben Hadj

Moshe Ben-Ezra

Martin Benning

Maitine Bergounioux

Michel Berthier
Jérémie Bigot

Isabelle Bloch

Radu Ioan Bot

Nicolas Boumal

Mireille Boutin

Laurence Boxer

Claire Boyer

Elie Bretin

Michael Breuss

Valentin E. Brimkov

Ricardo Cabral

Nathan Cahill

Jianfeng Cai

Xiaohao Cai

Yu Cao

Bruno Carvalho

Jan Cech

Pierre Chainais

Antonin Chambolle

Guillaume Charpiat

Christophe Charrier

Kunal Chaudhury

Caroline Chaux

Yunjin Chen

N. Chernov

Gregory Chirikjian

Krzysztof Ciesielski

David Coeurjolly

Bartomeu Coll

Tomeu Coll

Anne Collard 
Patrick Combettes

Laurent Condat

Marco Cuturi

Anders Bjorholm Dahl

Mauro Dalla Mura

Sven de Vries

Johan Debayle

Eric Debreuve

Etienne Decencière

Agnes Desolneux

Stephan Didas

Leo Dorst

Ulrich Eckhardt

Martin Ehler

Sameh Abdelwahab Nasr Eisa

Michael Elad

Ali Özer Ercan

Selim Esedoglu

Gabriele Facciolo

M. Felsberg

Carlos Fernandez-Granda

Jan Henrik Fitschen

Oliver Fleischmann

Michael Floater

Alessandro Foi

Friedrich Fraundorfer

Sébastien Gadat

Guillermo Gallego

Qi Gao

Edgar Garduño

Antonios Gasteratos

Andreas Geiger

Thierry Géraud

Aurobrata Ghosh

Jerome Gilles

Antonio Giraldo

Raja Giryes

Bastian Goldluecke

Ardeshir Goshtasby

Yann Gousseau

Gottfried Graber

Markus Grasmair

Nicola Greggio

Lewis Griffin

Jean-Philippe Groby

Mijail Guillemard

Onur Guleryuz

Weihong Guo

Rami Hagege

David Hammond

Xuli Han

Gloria Haro

Mattias Heinrich
A. Heyden

Moncef Hidane

Klaus Hildebrandt

Eckard Hitzer

Laurent Hoeltgen

Martin Holler

Byung-Woo Hong

Ming-Huwi Horng

Thomas Hotz

Xiaodi Hou

Zhanyi Hu

Maxime Ihuillier

Armin Iske

Arpit Jain

Ali Jamaludin

Marko Janev

D. Jeulin

Alexis Joly

Yong Chul Ju

Miyoun Jung

Irène Kaltenmark

Kenichi Kanatani

Myungjoo Kang

Sung Ha Kang

Chiu-Yen Kao

Lina Karam

Vladimir Katkovnik

Yossi Keller

Charles Kervrann

Dinh Khanh

Vladimir Kim

Clemens Kirisits

Nahum Kiryati

Valdimir Kolmogorov

Tat Kong

Simon Korman

Abdelouahed Kouibia

Michael Krause

Christian Kruschel

Stefan Kunis

Jacques-Olivier Lachaud

Andreas Langer

Stanislas Larnier

Carole Le Guyader

Karim Lekadir

Jan Lellmann

Jakob Lemvig

Vincent Lepetit

Olivier Lezoray

Hongdong $\mathrm{Li}$

Jingwei Liang

Aristidis Likas

Tony Lindeberg 
Alexander Lion

Shengjun Liu

Dirk Lorenz

Yifei Lou

Haiping Lu

Russell Luke

Julien Mairal

Petros Maragos

Antonio Marquina

P. C. H. Martens

Simon Masnou

Bogdan Matuszewski

Pierre Maurel

Loïc Mazo

P. Meer

Etienne Memin

Facundo Memoli

Jose Mennesson

Andrea Mennucci

Francois Meyer

Peyman Milanfar

Washington Mio

Jan Modersitzki

Michael Moeller

Lionel Moisan

Salvador Moll

Juan Monterde

R. Mukandan

Sungkwang Mun

Monika Muszkieta

Jim Nagy

Laurent Najman

Heiko Neumann

Mai Nguyen-Verger

Mads Nielsen

Mila Nikolova

Matteo Novaga

Alexander Oberdörster

Takayuki Okatani

P. J. Olver

M. Oskarsson

Georgios Ouzounis

A. Cengiz Oztireli

Nicolas Papadakis

Kostas Papafitsoros

Jaesik Park

Nicolas Passat

Vladimir Pavlovic

Kim Pedersen

Xingxuan Peng

Sergiy Pereverzyev

Benjamin Perret

Jean-Christophe Pesquet
Pascal Peter

Stefania Petra

Jean-Marc Peyrat

Luis Pizarro

Jorg Portegies

Alexander Powell

Tobias Preusser

Srikumar Ramalingam

Ronny Ramlau

Romain Raveaux

Michael Rieck

Paul Rodriguez

Jose Ronda

Martin Rumpf

Lars Ruthotto

Rayan Saab

Christophe Saint-Jean

David Salinas

Joseph Salmon

Mathieu Salzmann

Stephen Sangwine

Hayden Schaeffer

Christian Scheffer

Christian Schmaltz

Frank Schmidt

Mark Schmidt

Thomas Schuster

Simon Setzer

Chen Shen

Lixin Shen

Adrian Sheppard

Kaleem Siddiqi

Amit Singer

Chandan Singh

Marcelo Siqueira

K. Sivakumar

Isabelle Sivignon

Dejan Slepcev

Jon Sporring

Anuj Srivastava

Lefkimmiatis Stamatis

Carsten Steger

Gabriele Steidl

Tomas Suk

Hongpeng Sun

Ganesh Sundaramoorthi

Peter Sussner

Imants Svalbe

Anne Marie Svane

Paul Swoboda

Zygmunt Szpak

Hemant Tagare

Xue-Cheng Tai 
Hugues Talbot

Alexandru Tamasan

Sibel Tari

Daniel Tenbrinck

Barend ter Haar Romeny

I. R. Terol-Villalobos

Markus Testorf

Yonghong Tian

Akihiko Torii

Roberto Tron

Carole Twining

Corinne Vachier

Tuomo Valkonen

Andrea Vedaldi

Olga Veksler

Santiago Velasco Forero

Luminita Vese

Francois-Xavier Vialard

Rene Vidal

Sebastian Volz

Michael Vynnycky
Christian Wachinger

Junyan Wang

Rongrong Wang

I. Weiss

Martin Welk

You-Wei Wen

Thomas Widlak

Richard Wildes

Michael Wilkinson

Julian Wörmann

John Wright

Jin Xie

Xiaojing Ye

Xiaoming Yuan

Ido Zachevsky

Tieyong Zeng

Xiaoqun Zhang

Yinqiang Zheng

Wei Zhu

Zoran Zivkovic

Steven Zucker 